\title{
Observation of Protons and Light Nuclei with CALET: Analysis and Preliminary Results
}

\author{
Pier Simone Marrocchesi* (for the CALET Collaboration) \\ Dept. of Physical Sciences, Earth and Environment, University of Siena and INFN Pisa \\ Via Laterina 8, I-56100 Siena and Largo B. Pontecorvo, 3 I-56127 Pisa \\ E-mail: piersimone.marrocchesi@pi.infn.it
}

\begin{abstract}
The CALorimetric Electron Telescope (CALET) has been collecting science data on the JEM-EF external platform of the ISS since mid-October 2015. To date, more than 300 million triggers generated by high-energy charged particles and photons from space have been recorded. The instrument includes a two-layered Charge Detector (CHD) equipped with segmented scintillators to identify - via a measurement of their electric charge - individual elements from proton to iron and to detect trans-iron nuclei. Complementary charge and tracking information is provided by the electromagnetic Imaging Calorimeter (IMC), instrumented with individually read-out scintillating fibers, and followed by a thick homogeneous calorimeter (TASC). The whole calorimetric system provides an excellent energy and angular resolution and electron/proton discrimination of order $10^{-5}$. In addition to its primary goal to explore the electron spectrum in the energy region above $1 \mathrm{TeV}$, CALET can also study the cosmic-ray hadronic sector by contributing high precision measurements of the energy spectra, relative abundances and secondary-to-primary ratios of elements from proton to iron. Deviations from a simple power-law, as reported by CREAM, PAMELA and AMS-02 in the spectra of light nuclei are under study from a few tens of $\mathrm{GeV}$ to the multi- $\mathrm{TeV}$ region. In this paper, a progress report on the analysis and preliminary results relative to the observation of protons and light nuclei with CALET will be given. CALET is a space mission of the Japanese Aerospace Agency (JAXA) in collaboration with the Italian Space Agency (ASI) and NASA.
\end{abstract}

35th International Cosmic Ray Conference

10-20 July, 2017

Bexco, Busan, Korea

\footnotetext{
* Speaker.
} 


\section{Introduction}

CALET is a space mission led by the Japanese Space Agency (JAXA) with the participation of the Italian Space Agency (ASI) and NASA. It was launched on August 19, 2015 with the Japanese carrier H-II and delivered to the ISS by the HTV-5 Transfer Vehicle, where it was installed on the Japanese Experiment Module Exposure Facility (JEM-EF).

The primary science goal of CALET $[1,2]$ is to carry out high precision measurements of the electron spectrum with an accurate scan of the energy region already covered by previous experiments [3] and to extend it to to the unexplored region above $1 \mathrm{TeV}$. The electron spectral shape might reveal the possible presence of nearby sources of acceleration. With excellent energy resolution (better than $2 \%$ for electrons above $100 \mathrm{GeV}$, proton rejection capability $>10^{5}$ and low background contamination, CALET is searching for possible signatures of dark matter in the spectra of both electrons and gamma rays.

CALET has also started long term observations of light and heavy cosmic nuclei $[4,5]$ from proton to iron and is currently chasing trans-iron elements [6] up to $\mathrm{Z} \sim 40$ taking advantage of its capability to identify cosmic rays with individual element resolution and measure their spectral shape and relative abundance in the energy range from a few tens of $\mathrm{GeV}$ to several hundred $\mathrm{TeV}$. In particular, CALET is investigating the intermediate energy region from $200 \mathrm{GeV} / \mathrm{A}$ to $800 \mathrm{GeV} / \mathrm{A}$ where a deviation from a single power-law has been observed for both proton and helium spectra by CREAM [7, 8, 9], PAMELA [10, 11] and confirmed with high statistics measurements by AMS-02 [12] that reported a similar behavior also for $\mathrm{Li}$ and other light nuclei. CALET is performing an accurate scan of this energy region to verify the hypothesis of a progressive hardening of the proton spectrum by measuring its curvature. On the basis of a very preliminary analysis, in this paper we show that CALET is in a position to close the gap between the AMS-02 highest energy points and CREAM lowest points and to extend the reach of precision measurements to the multi- $\mathrm{TeV}$ region as well as for He and other light nuclei. A preliminary study of heavier nuclei with CALET is also reported at this conference [13].
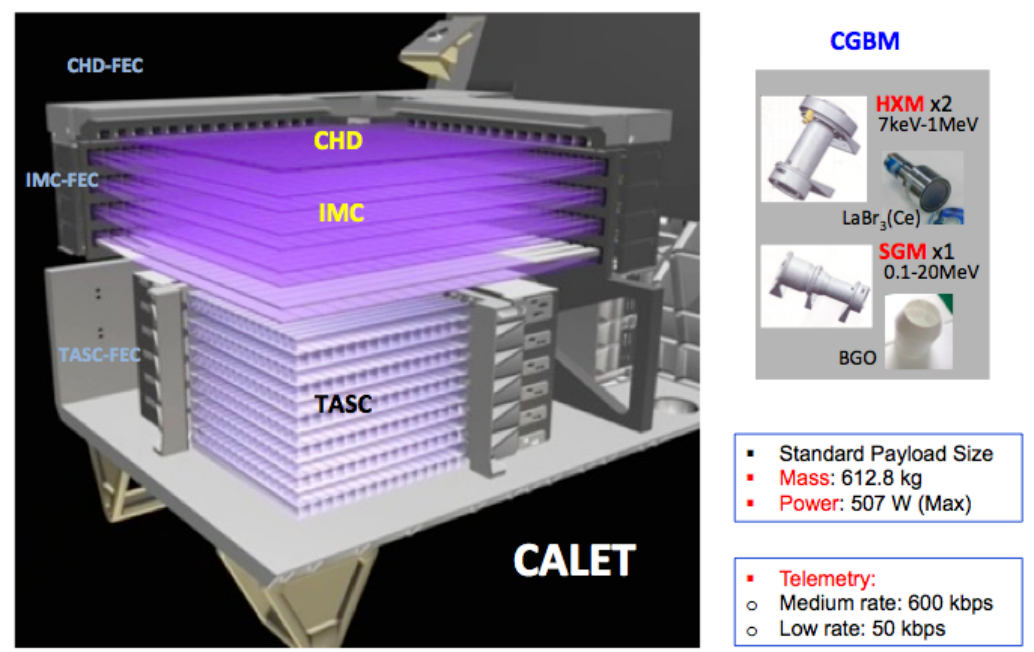

Figure 1: Layout of the CALET instrument and of the Gamma-Ray Burst Monitor (CGBM). 


\section{CALET (CALorimetric Electron Telescope)}

CALET (Fig.1) includes CAL, an all-calorimetric instrument designed to achieve a large proton rejection capability $\left(>10^{5}\right)$ with a fine grained imaging calorimeter (IMC) followed by a total absorption calorimeter (TASC). The overall thickness of CALET at normal incidence is 30 $\mathrm{X}_{0}$ and $\sim 1.3$ proton interaction length $\left(\lambda_{I}\right)$. The charge identification of individual nuclear species is performed by a two-layered hodoscope of plastic scintillators (CHD), positioned at the top of the apparatus, providing a measurement of the charge $\mathrm{Z}$ of the incident particle over a wide dynamic range $(Z=1$ to $\sim 40)$ with sufficient charge resolution to resolve individual elements $[14,15,16]$ and complemented by a redundant charge determination via multiple $\mathrm{dE} / \mathrm{dx}$ measurements in the IMC. The latter is a sampling calorimeter segmented longitudinally into 16 layers of scintillating fibers readout individually. It can image the early shower profile in the first $3 \mathrm{X}_{0}$ and reconstruct the incident direction of cosmic rays with good angular resolution $\left(0.1^{\circ}\right.$ for electrons and better than $0.5^{\circ}$ for hadrons) [2]. The TASC is a $27 \mathrm{X}_{0}$ thick homogeneous calorimeter with 12 alternate $\mathrm{X}$-Y layers of lead-tungstate (PWO) logs.

The geometrical factor of CALET is $\sim 0.12 \mathrm{~m}^{2} \mathrm{sr}$ and the total weight is $613 \mathrm{~kg}$. The instrument is described in more detail elsewhere $[1,4,17]$. Gamma-ray transients are detected by a dedicated Gamma-ray Burst Monitor (GBM).

\section{Data Analysis}

The preliminary analysis presented in this paper is based on flight data covering a period of 15 months of observation from December $1^{s t}, 2015$ to February $28^{\text {th }}, 2017$ and collected with a dedicated high energy shower trigger [17]. A live time fraction to total time close to $84 \%$ was achieved during this period [3]. The stability of the operations on the ISS and the continuous inflight calibrations of the instrument provided an excellent basis for accurate direct measurements of charged and neutral cosmic radiation. A detailed report of the calibration procedures and their achievements can be found elsewhere [17].

At the present stage of the analysis, the reconstructed events are accepted only within a fiducial region, known as Acceptance-A, limited to a geometric factor of $\sim 0.042 \mathrm{~m}^{2} \mathrm{sr}$ (about $40 \%$ of the total GF) where border effects (most notably shower lateral leakage) are not significant.

\subsection{Charge identification}

The identification of cosmic nuclei via a measurement of their charge is carried out in CALET with two independent subsystems that are routinely used to cross-calibrate each other: the CHD and the IMC. The latter, being equipped with individually readout scintillating fibers, has a suitable granularity to provide excellent tracking capabilities [18] and to sample the ionization deposits in each layer thereby providing a multiple $\mathrm{dE} / \mathrm{dx}$ measurement with a maximum of 16 samples along the track. The interaction point (IP) is first reconstructed [19] and only the $\mathrm{dE} / \mathrm{dx}$ ionization clusters from the layers upstream the IP are used. The charge value is evaluated as the truncatedmean of the valid samples with a truncation level set at $70 \%$. The non-linear response due to the saturation of scintillation light in the fibers is corrected for by fitting the light yield according to the halo model of $[20,21]$ whereby scintillation saturates at close distance from the primary track. Linearity is recovered at larger radial distance due to the secondary ionization from delta-rays 
injected in the halo all along the track. An expansion of the differential light yield $\frac{d L}{d x}$ as a function of the scintillator thickness $x$ in powers of $Z^{2}$ leads to the following parametrization [22] :

$$
\frac{d L}{d x}=\frac{A\left(1-f_{h}\right) Z^{2} \alpha}{1+B_{s}\left(1-f_{h}\right) Z^{2} \alpha+G Z^{4} \alpha^{2}}+A f_{h} Z^{2} \alpha
$$

where $f_{h}$ is the fraction of ionization in the halo; $B_{s}$ is related to the strength of the light quenching; $\alpha \sim 2 \mathrm{MeV} / \mathrm{gr} \mathrm{cm}^{-2}$ for plastic scintillators, $\mathrm{Z}$ is the atomic number, $\mathrm{G}$ is numerically relevant only for heavy nuclei and $\mathrm{A}$ is an overall normalization constant.

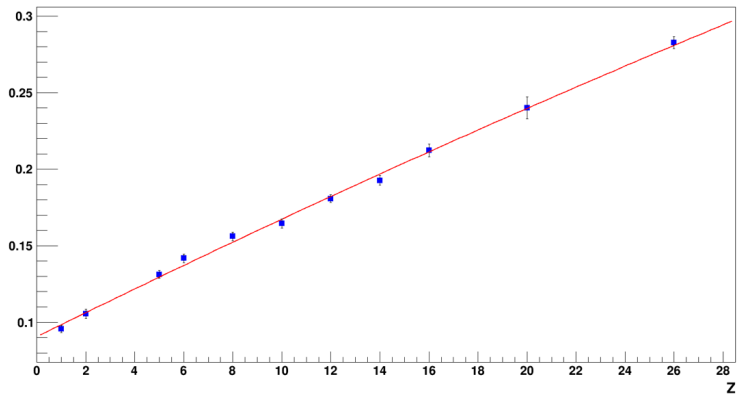

(a)

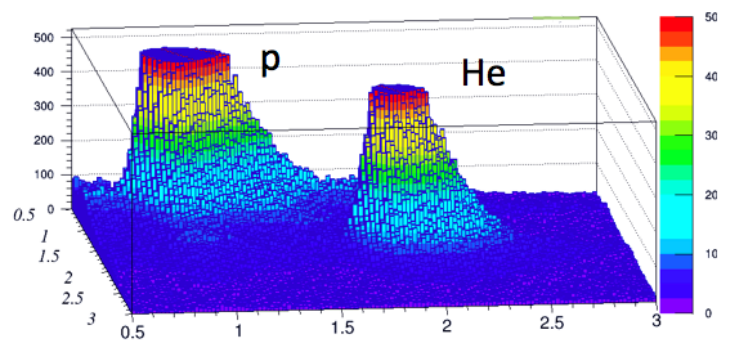

(c)

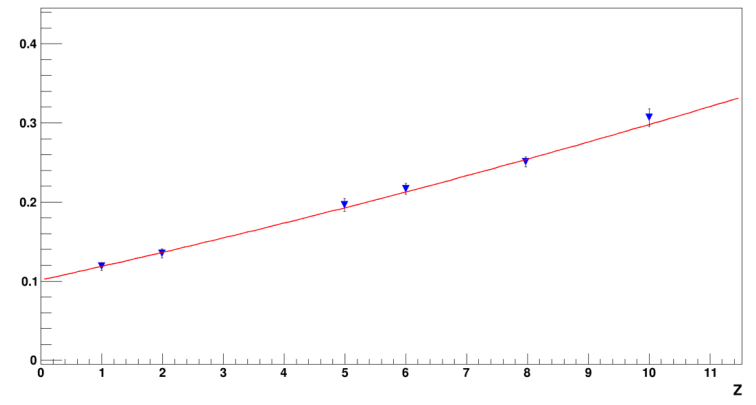

(b)

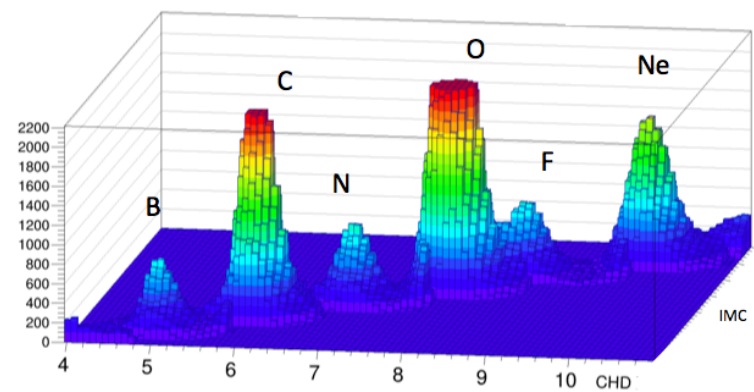

(d)

Figure 2: (a) Charge resolution (in units of the elementary charge) vs. atomic number $\mathrm{Z}$ for the two CHD layers combined; (b) IMC charge resolution for a selection of light elements; (c) Charge distribution of proton and helium candidates in the Z-plane (see text); (d) Charge distributions for B, C, N, O, F, Ne candidates in the Z-plane. The charge peaks of the most abundant elements have been chopped off in the picture to improve the visibility of the less abundant ones.

Charge resolution After application of position and time-dependent calibrations and corrections for each CHD paddle [17] (e.g: gain uniformity, light yield as a function of distance from the PMT, corner-clipping effects, etc.) and of the aforementioned correction for the non-linear response of the scintillator, the information from the two CHD layers is combined into a single charge estimator. Consistency between the charge assignment from two CHD layers is required within 20\%. The overall CHD charge resolution (in $\mathrm{Z}$ units) is then plotted in Fig.2(a) showing a linear increase as a function of the atomic number from $<0.1$ for protons to $\sim 0.3$ for iron.

For the IMC, although the photostatistics available at single fiber level is about one order of magnitude lower than a single CHD layer, the charge measurement - thanks to the multiple sampling - can achieve an adequate performance as shown in Fig.2(b) where the IMC charge 
resolution is plotted as a function of the atomic number $\mathrm{Z}$ after restoration of a linear response as explained above. An adequate separation power is provided for instance for boron and carbon with a charge resolution of $\sim 0.14$ in the CHD and $\sim 0.2$ in the IMC.

Combined charge identification with CHD and IMC - By correlating the two charge measurements, as in the example shown in Fig.2(d) where the IMC charge is plotted versus the CHD charge (hereafter referred to as Z-plane), well separated charge peaks emerge on top of a low and flat background for light nuclei candidates from boron to neon. This is also true for the lightest elements proton and helium as shown in Fig.2(c). Under these conditions a clean charge separation is possible allowing for a robust rejection power of He candidates in the selected proton sample as explained in the following.

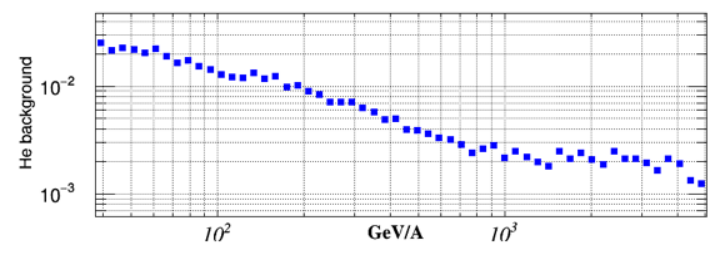

(a)

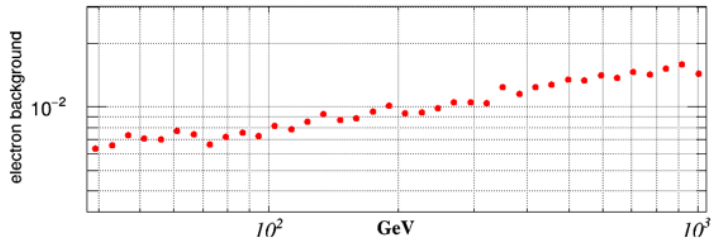

(b)

Figure 3: (a) Fractional background contamination of He (blue filled squares) up to $5 \mathrm{TeV} / \mathrm{A}$ and (b) Fractional background contamination of electrons (red filled circles) in the selected proton sample vs. Energy (GeV) up to $1 \mathrm{TeV}$.

\section{Preliminary Proton Analysis}

Due to the limited equivalent thickness of $1.3 \lambda_{I}$ of the IMC+TASC calorimeter module, only $\sim 80 \%$ of protons interact on average in CALET. The High-Energy Trigger (HET) efficiency ${ }^{1}$ rises very slowly from a threshold close to $7 \mathrm{GeV}$ and reaches $10 \%$ at $50 \mathrm{GeV}$ which is the lowest energy point we have included in the present analysis. Neither geomagnetic nor solar modulation effects play a significant role above this energy.

Charge-selection and Helium background - Charge selection of proton events is performed by application of an elliptical cut in the Z-plane. This cut has been studied to allow for a mild residual energy dependence of the position of the CHD proton charge peak most probably related to the energy increase of backscattering from the TASC. A residual fractional contamination of helium falling below the percent level above $200 \mathrm{GeV}$ is shown (blue squares) in Fig.3(a) and subtracted.

Electron background - Rejection of electrons is at present achieved by using a cut on the fraction of energy deposited in the bottom layer of the TASC with respect to the total energy. The proton efficiency for this cut is $\sim 90 \%$ with a mild dependence on energy. Most of the electron background is below $50 \mathrm{GeV}$ therefore there is no need to introduce a more severe selection at the expense of proton efficiency. Above $50 \mathrm{GeV}$ the remaining contamination of electrons in the proton sample (red filled circles) in Fig.3(b) is close to the percent level and it is subtracted.

Energy Unfolding - Differently from electrons, the measured energy of protons is significantly lower than the primary particle's energy (typically $\sim 40 \%$ ) and smeared by the

\footnotetext{
1 a dedicated Low Energy trigger (LE) is in place to study electron energies down to about $1 \mathrm{GeV}$.
} 
calorimeter resolution for hadronic showers causing a migration of events from a given energy bin into the nearby ones. A standard technique to recover the true value of the primary particle's energy is to construct, using MC data, an energy overlap matrix whereby each matrix element $\alpha_{i j}$ and normalization factor $\mathrm{n}_{j}$ are weighted when the MC primary energy falling into the $\mathrm{i}$-th bin leads to a reconstructed energy deposit in bin j. Let $A_{i j}=\frac{\alpha_{i j}}{n_{j}}=$ normalized overlap matrix elements; $\varepsilon_{i}=$ total efficiency in i-th bin; $\beta_{j}=$ background contamination in $\mathrm{j}$-th bin; $\mathrm{M}_{j}=$ number of (weighted) events measured in $\mathrm{j}$-th bin; $\mathrm{M}_{t o t}=$ sum of the $\mathrm{M}_{j}$ within the energy range of the measurement. The unfolded number $\mathrm{N}_{i}$ of (weighted) events in i-th bin can then be written as:

$$
N_{i}=\frac{1}{\varepsilon_{i}} \sum_{j=1}^{n} A_{i j}\left(M_{j}-\beta_{j} M_{t o t}\right)
$$

A graphical representation in false colors of the overlap matrix $\mathrm{A}_{i j}$ is shown in Fig.4 with bin numbers from 1 to 100 on both axes. The width of the diagonal line is representative of the energy spread due to the finite resolution of the calorimeter. After background subtraction, energy unfolding is applied to convert measured energy into primary one. Energy dependent corrections for efficiencies and acceptance are then applied to the selected proton candidates.

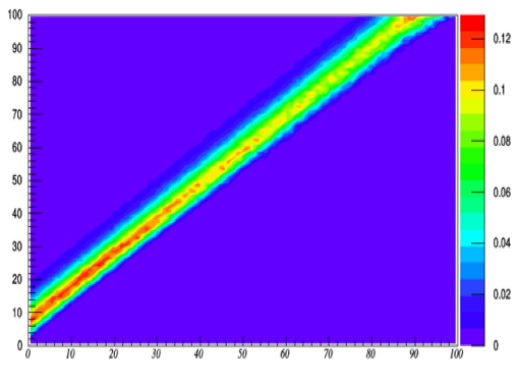

Figure 4: Representation in false colors of the values of the normalized energy overlap matrix elements $A_{i j}$ (energy bin indices on both axes)

Efficiency and Acceptance Corrections The main efficiency corrections (trigger, tracking, charge selection, electron-ID) are individually studied as a function of the MC true energy using three different MC simulation packages: FLUKA, EPICS and GEANT4. Tracking efficiency reaches a $\sim>95 \%$ plateu at $1 \mathrm{TeV}$ while it has a value of $\sim 90 \%$ at $100 \mathrm{GeV}$ with room for improvement at lower energies. Charge selection efficiency is almost flat at $95 \%$ above $50 \mathrm{GeV}$ slowly decreasing to $\sim 90 \%$ at $10 \mathrm{TeV}$. The electron rejection efficiency is $\sim 93 \%$ at $100 \mathrm{GeV}$ and $\sim 90 \%$ at $10 \mathrm{TeV}$. The HET trigger efficiency (normalized to all events in acceptance including non interacting protons) is $\sim 40 \%$ at $1 \mathrm{TeV}$. The probability of a wrong assignment of the acceptance type, as provided by the reconstruction of the track and of the shower axis, is taken into account in the analysis.

Systematic uncertainties Sources of systematic uncertainty related to the absolute normalization of the flux include geometrical acceptance, absolute energy scale, trigger efficiency, track reconstruction, charge selection, electron identification, background modeling, live time measurement and long-term stability of the detector. Systematics related to the spectral shape and spectral index dependence include the assessment of the energy scale and the energy dependence of efficiency corrections. This is of particular relevance for the trigger efficiency of protons as it 


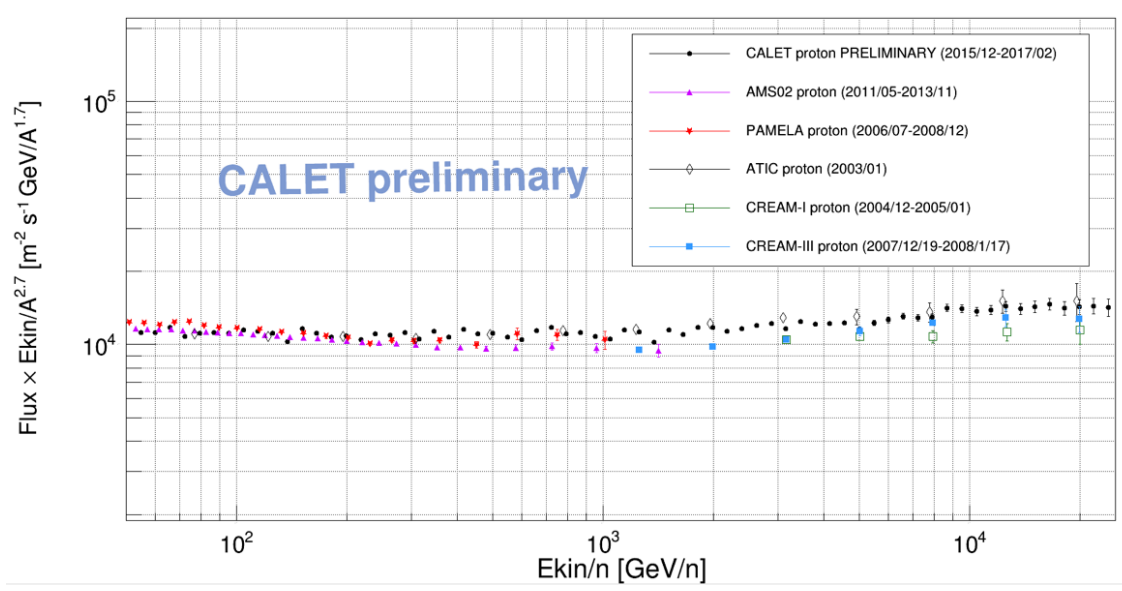

(a)

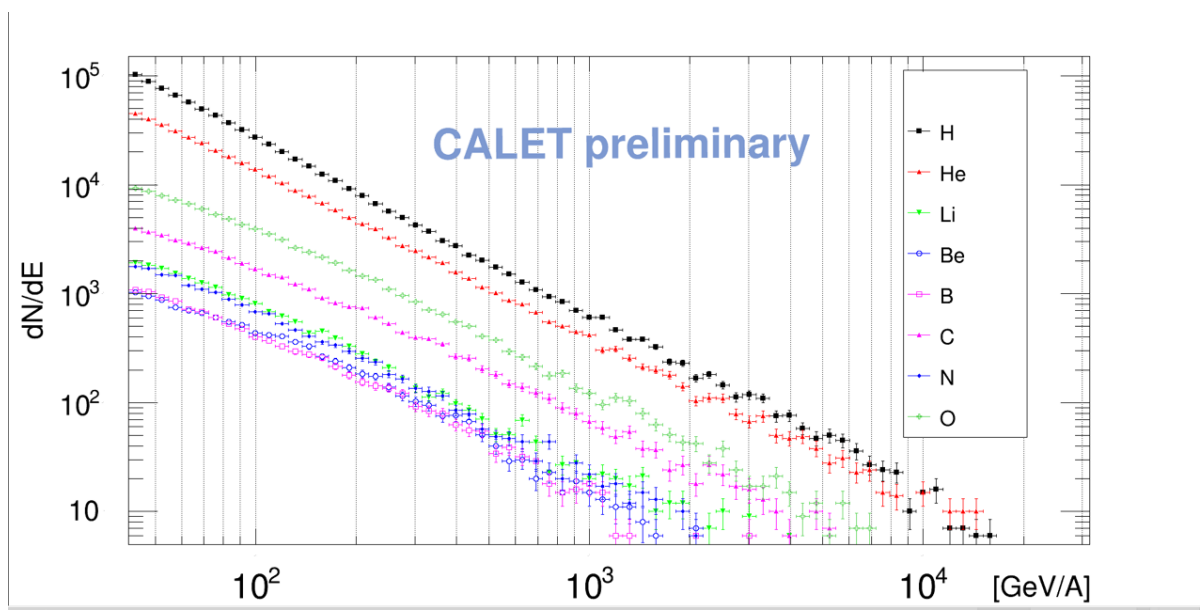

(b)

Figure 5: (a) Preliminary proton flux $\times \mathrm{E}^{2.7}$ vs. particle kinetic energy $\mathrm{E}$ in $\mathrm{GeV}$ from $50 \mathrm{GeV}$ to $22 \mathrm{TeV}$. Only statistical errors are shown; (b) Preliminary energy distribution $\mathrm{dN} / \mathrm{dE}$ in kinetic energy per nucleon [GeV/A] for light elements from proton to oxygen. Both plots refer to a sub-sample of the available data collected in a restricted fiducial volume.

increases with energy. Application of the standard techniques to investigate the stability of the measurement by scanning the parameter space is underway. In particular, the differences in the templates distributions from the three simulation packages are a powerful tool to disentagle the various sources of systematics.

The preliminary CALET data points for the proton differential flux multiplied by $\mathrm{E}^{2.7}$ vs. particle kinetic energy E are shown (black filled circles) in Fig.5(a) where the error bars refer to statistical errors only. A partial compilation of proton data include the recently published result from CREAM-III [9]. The assessment of the systematic uncertainties in the proton analysis (not presented here) will improve in time along with the steady increase of the available statistics and more refined understanding of the instrument.

\section{Light nuclei}

The analysis of the absolute and relative fluxes of light elements is underway. It requires, for 
each chemical species under study, the subtraction of the residual background contamination in the selected sample. The main sources of background include: (i) charge changing interactions in the apparatus (mainly by nuclear spallation); (ii) wrong charge assignment due to the presence of backscattered radiation from the calorimeter. The former typically produces a migration of event candidates toward lower atomic numbers while the latter works in the opposite direction because of the extra energy deposits collected along the track on top of the ionization generated by the primary particle. The measurement of the relative elemental abundances requires a correction for the above effects. This is usually encoded into an array that contains the expected transition probabilities among the different nuclei as derived from the MC simulations. Also, energy deconvolution has to be carried out. At the present preliminary stage of the analysis we only show the $\mathrm{dN} / \mathrm{dE}$ distributions of light nuclei candidates after a simple data pre-selection requiring a high energy trigger (HET) and a well reconstructed track inside Acceptance-type A. Charge selection is then performed in the IMC vs CHD charge plane by applying a circular cut of radius 0.8 (in $\mathrm{Z}$ units) around the nominal charge $\mathrm{Z}$ for each element with $2<\mathrm{Z}<11$. The selected candidates from proton to oxygen are plotted in Fig.5(b) as a function of the reconstructed calorimetric energy (in GeV/A units).

\section{Conclusions}

A preliminary analysis based on a sub-sample of the data collected during the first 15 months of observation on the ISS has shown the capability of CALET to extend by more than one order of magnitude the energy reach of the direct measurements of the proton spectrum provided by magnetic spectrometers and to investigate the spectra of light nuclei. The so far excellent performance of CALET and the outstanding quality of the data suggest that a 5-year observation period might provide a wealth of new interesting data.

\section{References}

[1] S. Torii et al., Proc. 33rd ICRC, 245 (2013)

[2] S. Torii et al., Proceedings of Science (ICRC2015) 581 (2015)

[3] Y. Asaoka et al., Proc. of the 35th ICRC (Busan, Korea), this conference (2017)

[4] P.S. Marrocchesi et al., Nucl. Instr. and Meth. A 692, 240âĂŞ-245 (2012)

[5] P.S. Marrocchesi, Charged Cosmic Rays: a Review of Balloon and Space Borne Measurements arXiv:1704.00304 [astro-ph.HE] (2017)

[6] B.F. Rauch, Pos(ICRC2015) 627

[7] H. S. Ahn et al., Astrophys. J. 714, 89-93 (2010)

[8] Y. S. Yoon et al., Astrophys. J. 728, 122 (2011)

[9] Y. S. Yoon et al., arXiv:1704.02512v1 [astro-ph.HE] (2017)

[10] O. Adriani, et al., Science 332, 69 (2011)

[11] O. Adriani, et al., Astrophys. J. 765, 91-98 (2013)

[12] M. Aguilar et al., Phys. Rev. Lett. 114, 171103 (2015)

[13] Y. Akaike et al., Proc. of the 35th ICRC (Busan, Korea), this conference (2017)

[14] Y. Shimizu et al., Proc. 32nd ICRC, 898 (2011)

[15] P.S. Marrocchesi et al., Nucl. Instr. Meth. A 659, 477-483 (2011)

[16] S. Torii et al., Nucl. Instrum. Meth. A 630, 55 (2011)

[17] Y. Asaoka et al., Astropart. Phys. 91, 1 (2017)

[18] P. Maestro and N. Mori, Proc. of the 35th ICRC (Busan, Korea), this conference (2017)

[19] P. Brogi et al., Proc. of the 34th ICRC (The Hague, Netherlands), 595.

[20] R. Dwyer et al., Nuclear Instruments and Methods in Physics Research A242 171-176 (1985)

[21] G. Tarle et al., Nuclear Instruments and Methods in Physics Research B6 504-512 (1985)

[22] F. Stolzi, PhD thesis - University of Siena (in preparation) 